\title{
Dermatitis de pabellón auricular por picadura de jerjeles. Una entidad clínica diferente no descrita
}

\author{
Dermatitis of the pinna by puncture of the black fly: \\ A different clinical disease not yet described
}

\author{
Alejandro Peña M¹.
}

\begin{abstract}
RESUMEN
Introducción: La picadura de jerjeles o simúlidos de la cara anterior del pabellón auricular presenta características clínicas diferente a las picaduras del mismo insecto en la piel del resto del cuerpo y requiere también un tratamiento diferente. Estos hechos no han sido descritos en la literatura.

Objetivos: El objetivo es detallar los síntomas y signos de estas picaduras y las causas de las diferencias clínicas y de su tratamiento.

Material y método: Para ello se hace el estudio y análisis de 6 pacientes aquejados por picaduras de jerjeles en la cara anterior de la oreja. Se investiga la biología de estos simúlidos. Finalmente se hace una revisión de la histología de la piel que recubre el cartílago auricular.

Resultados: El síntoma principal de todos los pacientes fue un intenso dolory edema de la oreja que les impedía dormir. La razón principal de estos síntomas es la íntima adhesión del dermis al cartílago auricular y que forma el pericondrio de éste, además de las múltiples toxinas salivales del insecto que tienen propiedades anticoagulantes, vasodilatadoras, e inhibidoras de la inmunidad celular.

Conclusiones: Las plagas de jerjeles y sus picaduras serán cada vez más numerosas en Chile debido al calentamiento global, la destrucción de los ecosistemas por el hombre que han ido eliminando sus enemigos naturales y la contaminación de la casi totalidad de ríos y lagos de nuestro país. Para el tratamiento de las picaduras de jerjeles del pabellón auricular es necesario recurrir al uso de corticoesteroides en dosis altas y por períodos breves de menos de una semana.
\end{abstract}

Palabras claves: Picaduras de jerjeles, black-fly punctures, díptera simuliidae punctures.

\section{ABSTRACT}

Introduction: Puncture of the black fly or dipteral simuliidae on the external surface of the ear auricle shows clinical characteristics which differ from the puncture of this same insect on other different parts of the body. Thus, it requires a different treatment, as

1 Médico Otorrinolaringólogo. Facultad de Medicina. Universidad Católica del Maule. 
well. These facts have not been clinically described yet, therefore, there is no literature available on this topic.

Aim: To describe symptoms and signs of this disease.To outline the causes of the clinical differences and medical treatment.

Material and method: Study and analysis of six patients suffering from puncture of black fly on the surface of ear auricle are carried out. The biology of these insects is studied. A revision of the histology of the skin of the ear auricle is made

Results: The main symptoms experimented by patients suffering from black fly puncture are: excruciating pain and severe edema on the auricle area. The pain even prevents them from sleeping. The main cause of these symptoms is that the skin on the external surface of the auricle is firmly attaches to the underlying cartilage. Besides, the countless toxins inoculated in the saliva of the fly contain not only anticoagulant agents but also produce vasodilatation of capillaries and inhibit cellular immunity.

Conclusions: The black fly plague and its infectious and painful puncture will be on the increase in Chile due to: global warming; elimination of the natural predators of this insect; man induced pollution of the majority of lakes and rivers in the country. The medical treatment for black fly puncture indicates corticosteroids in high dose for a short period of time (under a week).

Key words: Black fly; black fly puncture; diptera simuliidae puncture.

\section{INTRODUCCIÓN}

La dermatitis del pabellón auricular provocada por la picadura de jerjeles o petros en la cara anterior 0 externa de la oreja, presenta características clínicas especiales e indicaciones terapéuticas que la diferencian de las reacciones inflamatorias provocadas por estos simúlidos en otras partes del cuerpo y curiosamente no descritas o ignoradas en la literatura médica científica ${ }^{1}$.

\section{OBJETIVOS}

Los propósitos u objetivos del presente estudio es conocer las razones o motivos de las características clínicas diferentes que muestran las picaduras de jerjeles en la cara anterior del pabellón auricular y que obligan a terapéuticas más potentes o intensas, estas preguntas se dilucidan a través del estudio y análisis de 6 casos clínicos, y el estudio de la biología de estos simúlidos.

\section{MATERIAL Y MÉTODO}

1. Casos clínicos: El estudio se basa en la descripción de los efectos de estas lesiones en seis pacientes aquejados por la picadura de jerjeles o petros en la piel que recubre el cartílago auricular de la cara anterior 0 externa de la oreja. Todos ellos tenían antecedentes clínicos, síntomas y signos similares. Se trataba de pacientes adultos jóvenes, entre $18 \mathrm{y}$ 40 años de edad, de sexo masculino.

Todos ellos consultaron el mes de enero, que se caracterizó por ser el mes más caluroso de las últimas décadas en la región del Maule, Chile.

También todos ellos, tenían o mostraban picaduras de jerjeles en el resto del cuerpo, extremidades o tórax, que les provocaban prurito intenso alrededor de la zona picada por los dípteros.

La totalidad de ellos habían sido picados durante el día y a pleno sol, en zonas rurales cubiertas de vegetación de árboles y arbustos, cercanas a ríos 0 arroyos, uno mientras pescaba truchas, otro en el jardín de su casa y, los otros cuatro en zonas de camping.

El conjunto de ellos presentaron síntomas similares, primero ardor y prurito intenso en la zona de la picadura con formación de una pápula en la zona en menos de 30 minutos, para después transformarse a las pocas horas, en un dolor progresivo en intensidad y persistente en la zona afectada y que no cedía a los antihistamínicos de segunda generación (desloratadina y levocetirizina), y con un alivio parcial y temporal con los 
AINES de consumo común (ibuprofeno, diclofenaco, ketorolaco etc).

El motivo principal de la consulta de todos ellos, era la dificultad de conciliar el sueño pues el dolor aumentaba al apoyar la cabeza sobre la almohada al estar en decúbito lateral con la oreja afectada hacia abajo.

Todos ellos habían sido picados por jerjeles en otras temporadas y conocían los síntomas.

2. Examen físico: El examen físico del pabellón auricular afectado ofrecía características similares: Cara anterior del pabellón auricular muy edematosa, con calor local, intensamente enrojecida, muy sensible a la palpación especialmente en las estructuras más prominentes de la oreja, el hélix y antihélix. Ninguno de ellos tenía fiebre o había tenido fiebre. Tampoco se palpaban adenopatías subauriculares, ni retro ni preauriculares. En cuatro de ellos se podía observar por medio de una lupa en un solevantamiento papuloso el punto de la picadura.

3. Biologia de los simúlidos o jerjeles (Diptera simuliidae) ${ }^{1-3}$. Los simúlidos conocidos en las zonas rurales de nuestro país como jerjeles o petros (black flies), son dípteros simúlides de distribución universal, pero particularmente abundantes en las zonas tropicales y subtropicales.

Constituyen una enorme familia de insectos, subdivididos en 4 familias, 5 tribus y 59 géneros. Se han identificado en el mundo alrededor de 1.300 especies, y en Chile 30 de ellas.

En el estado adulto, son moscas pequeñas de 1,2 a $5 \mathrm{~mm}$, habitualmente negras en Chile, de patas cortas, alas anchas y un aparato bucal

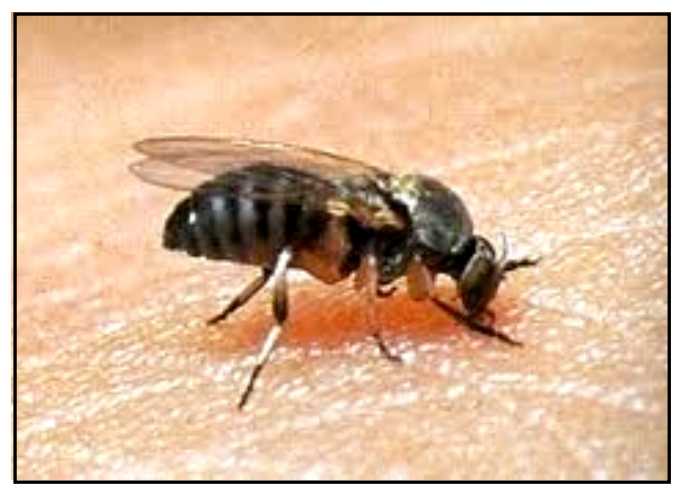

Figura 1. terminado en un estilete con el cual rasgan la piel (Figura 1). La cantidad de generaciones anuales varía según la temperatura ambiental de 4 a 20 por año. Sus huevos son de un diámetro de 0,18 a 0,46 $\mathrm{mm}$.

Son picadores diurnos. Los jerjeles hembras, son considerados las plagas más importantes para los seres humanos y los animales ${ }^{1}$, pues son hematófagos y requieren alimentarse de animales de sangre caliente, particularmente de mamíferos y aves, para poder desarrollar y desovar huevos fértiles. En cambio los machos se alimentan del néctar o la savia de las plantas.

Son vectores de muchas enfermedades, virales, bacterianas, protozoarias y por nematodes (de la familia de los áscaris) especialmente en el África sub-sahariana, Amazonas y selvas de Centroamérica y Asia. Al respecto la enfermedad más conocida y grave transmitida por los jerjeles (específicamente el Similium yahense y el Simulium guianense), es la llamada onchocerciasis 0 volvulosis o ceguera de los ríos tropicales, 0 enfermedad de Robles, llamada así en homenaje al médico guatemalteco Dr. Rodolfo Robles Valverde (1878-1939), quien descubrió el ciclo biológico extraordinariamente complejo de este nematode, parásito de los simúlides ${ }^{4}$, pues no es el onchocerca volvulus el causante de la ceguera sino una bacteria del nematode (endosimbiótica) llamada Wolbachia pipientis que se libera en la sangre de los pacientes picados por los jerjeles al morir el vermis y provoca la reacción inflamatoria ocular con eventual ceguera secundaria. La pérdida de visión que origina la volvulosis es actualmente la segunda causa mundial de ceguera infecciosa después del tracoma ${ }^{4}$. La OMS estimaba que el año 2008, existían 18 millones de personas infectadas por el parásito y 300.000 ciegos a consecuencia de la onchocerciasis.

Los jerjeles adultos se agrupan en enjambres enormes en la vecindad de ríos pequeños 0 arroyos con corrientes fuertes y rápidas pues requieren aguas muy bien oxigenadas para el desarrollo larval. Allí cada insecto hembra pone alrededor de 200 a 500 huevos. La larva una vez eclosionado el huevo, se fija a alguna estructura rocosa o planta a través de una estructura anal que funciona como ventosa, alimentándose de otras larvas o detritus celulares. El ciclo de vida acuática depende funda- 
mentalmente de la temperatura del agua y de la cantidad de alimentos, pero habitualmente es muy rápido de unos pocos días o unas pocas semanas. Los enemigos naturales de estas larvas son los pequeños peces, pequeños crustáceos, los batracios y las aves zancudas perseguidos y casi exterminados en nuestro país por el hombre y es la razón por la cual estos dípteros son cada vez más abundantes $^{5-9}$, y en Arica se ha convertido en un grave problema sanitario, lo cual unido a la pesca indiscriminada, el calentamiento global, y la contaminación de casi todos los lagos y ríos de Chile empeora la situación.

Desde el estado larval al estado de pupa pueden pasar de 2 a 28 días, dependiendo como se dijo previamente de la temperatura del agua. Los huevos dipausan durante el fin de verano, el otoño y el invierno, siendo extraordinariamente resistente, por ejemplo en las estepas siberianas son capaces de resistir intensos fríos, para después desarrollarse como imagos 0 insectos perfectos el siguiente verano.

Una vez desarrollado el insecto adulto y terminado su ciclo acuático, los jerjeles pueden volar entre 12 a $18 \mathrm{Km}$ o más, dependiendo de la fuerza del viento, ubicándose los enjambres en zonas de matorrales 0 árboles pequeños, allí los machos se alimentan del néctar y las hembras de la sangre de aves y mamíferos.

El ciclo de vida del insecto adulto es corto de 14 a 21 días. Como se señaló previamente los patrones climáticos estacionales afectan los patrones de abundancia y desarrollo de los simúlidos.

La eliminación de estas plagas en Chile ha sido extraordinariamente difícil, al haber el hombre eliminado sus enemigos naturales. La mayor experiencia es la de Arica, que debe estar constantemente pulverizando insecticidas en los valles ariqueños, con éxitos cortos y temporales, unido a la toxicidad de estos insecticidas, para otros insectos beneficiosos, aves, mamíferos y para también el ser humano $0^{8,9}$.

Países con muchos más recursos económicos como EE.UU. gastan enormes cantidades de dinero para su control, también con un éxito parcial, a pesar que es un país desarrollado con una población mucho más culta que tiene mayor respeto por la naturaleza ${ }^{1}$.

\section{RESULTADOS}

Como se señaló en la introducción las picaduras de estos insectos en el pabellón auricular presentan características particulares no descritas en los textos de estudio de la especialidad.

La razón principal de esta diferente reacción inflamatoria de la cara anterior del pabellón auricular es histológica. La piel que recubre el esqueleto cartilaginoso de la oreja en su cara anterior es muy delgada, carece de hipodermis o de tejido subcutáneo, y la segunda capa o dermis se condensa para constituir el pericondrio, de manera que ella está íntimamente adherida al cartílago y explica, por ejemplo, la gravedad de los hematomas traumáticos subpericóndricos de la oreja que deben ser drenados urgentemente para evitar la isquemia del cartílago y su eventual necrosis.

En el caso de la reacción alérgica-inflamatoria producida por la picadura de los jerjeles, el edema provoca un despegamiento del dermis con gran dolor y que impide a los pacientes apoyar la cabeza sobre la oreja en la almohada, y conciliar el sueño.

Un mecanismo similar del dolor es lo que sucede con la otitis externa difusa en la que los pacientes acusan un intenso dolor, pues aquí también la piel está adherida al periosto de la parte ósea del conducto auditivo externo

Para picar la piel los jerjeles poseen un aparato bucal parecido un estilete con el cual introducen saliva junto con chupar la sangre.

En la saliva de los jerjeles se han identificado una serie de toxinas antihemostáticas que son comunes a todas las especies. Se ha identificado una toxina llamada apirasa que inhibe la agregación plaquetaria; una proteína antitrombina que potencia la actividad antiagregante plaquetaria de la apirasa; una toxina vasodilatadora que facilita el aflujo de la sangre al sitio de la picadura y que explica el gran enrojecimiento del pabellón auricular al ser picado por estos insectos; se ha pesquisado además, en algunas especies, una toxina salival que afecta la respuesta inmunocelular y la producción de citoquinas y que explicaría la diseminación del nematode en la enfermedad de Robles 0 ceguera de los ríos tropicales ${ }^{10-12}$.

En la picadura de las orejas por los jerjeles, la inflamación alcanza su máxima intensidad al quinto día, para después ir remitiendo lentamente al cabo de dos semanas aproximadamente. 
Diagnóstico clínico: El diagnóstico se basa, primero, en los antecedentes de haber permanecido al sol en zonas rurales cercanas a ríos o arroyos, segundo en el examen clínico que permite observar el pabellón afectado intensamente rojo, edematoso y muy doloroso a la palpación, y la presencia de una pápula y el punto de la picadura, esto último más visible por medio de una lupa o por el microscopio quirúrgico y tercero la presencia eventual de picaduras en otras partes del cuerpo que ayudarían a confirmar el diagnóstico.

Diagnóstico diferencial: El diagnóstico diferencial podría plantearse con picaduras de otros insectos, mordedura de arañas, celulitis facial con diseminación auricular, erisipela con extensión auricular, herpes zoster ótico en el período preeruptivo, dermatitis de contacto, policondritis recidivante. Una buena anamnesis y un cuidadoso examen permitirá a cualquier especialista descartar estas opciones diagnósticas ${ }^{13-16}$.

Tratamiento: El tratamiento con antihistamínicos de la picadura de jerjeles de pabellón auricular, según experiencia personal no es útil. Los AINES disminuyen sólo parcialmente la inflamación y temporalmente el dolor.

Definitivamente parecieran ser los corticoides la terapia más útil para disminuir el edema y el dolor. En tal sentido el uso de corticoides en dosis altas $1 \mathrm{mg} / \mathrm{kg}$ al día y por períodos cortos de 5 días sería la mejor opción asociado o no a tratamiento local con pomada de corticoides por ejemplo, la mometasona.

\section{ANÁLISIS DE RESULTADOS}

Es este un estudio según los cánones de la Medicina Basada en Evidencias de nivel 4, es decir una investigación clínica descriptiva no experimental, que no requiere de un grupo control motivo por el cual no permite probar causa-efecto, pero sí puede documentar potencial buena información para casos particulares o específicos. A este respecto las recomendaciones de estudios de nivel 4 , son consideradas en el Grado C, de la Medicina Basada en Evidencias ${ }^{16}$.

\section{DISCUSIÓN Y CONCLUSIONES}

Del estudio de los casos clínicos expuestos y del conocimiento biológico de los Diptera simuliidae, es posible sacar las siguientes conclusiones

1. La picadura de los jerjeles en la cara anterior del pabellón auricular ofrece características diferentes a las picaduras de la piel de otras partes del cuerpo, particularmente el intenso dolor, y que no está descrito en los textos de estudio, ni en la literatura médica investigada.

2. Este dolor es debido a las características histológicas de la piel que recubre el cartílago auricular anteriormente pues no tiene hipodermis y la dermis constituye el pericondrio del cartílago de manera que su despegamiento por el edema inflamatorio es el origen del molesto síntoma.

3. Los jerjeles son una plaga en constante crecimiento debido al calentamiento progresivo de la tierra, con veranos cada vez más cálidos en Chile, a la eliminación alarmante de sus enemigos naturales por acción del hombre, en especial los peces de agua dulce, los pequeños crustáceos, las aves zancudas, y los batracios, rompiendo así el delicado equilibrio ecológico, a lo que se une la contaminación de casi todos los ríos y lagos de nuestro país por ignorancia, y desprecio del cuidado de la naturaleza del hombre.

4. El control de estas plagas es extremadamente difícil, siendo los insecticidas sólo de utilidad parcial y transitorio, agregado a los peligros que conlleva su uso en los otros seres vivos.

5. El tratamiento de este tipo de picaduras, debe ser diferente al habitual pues los antihistamínicos no son útiles y los AINES controlan sólo parcialmente el dolor y la inflamación. Son los corticoides orales y tópicos la herramienta más útil en su tratamiento.

\section{BIBLIOGRAFIA}

1. Dale E William. Plagas pest, médicoveterinarias.Simúlidos o jerjeles. Disponible en: www.lamolina.edu.pe/profesores/wdale/ent_vet/ 2/. Consultado el 23-02-2012.

2. Coscaron Sixto. Las especies de jerjeles (simuliidae díptera insecta) en la zona de Arica. 
Rev Departamento de Agricultura Universidad del Norte 1976; 4: 25-34.

3. Henry Abel, Bobadilla Dante. Los jerjeles (díptera simuliidae) de Arica, Chile. Rev Per Ent 1981; 24(1): 117-21.

4. Onchocerciasis. Disponible en: http:// en.wikipedia.org/wiki/onchocerciasis. Consultado el 23-02-2012.

5. Diario La Estrella de Arica: 23-10-2001; Buscan fondos para combatir los jerjeles.

6. Diario La Estrella de Arica:16-01-2004; La cuarta plaga de jerjeles espanta a los turistas.

7. Diario La Estrella de Arica:03-01-2006; Vecinos molestos por los jerjeles.

8. Boletín Intendencia de Arica y Parinacota: 0801-2008; Programa de control de jerjeles listo para su ejecución.

9. Diario El Morrocotudo de Arica: 06-06-2011; Dígale adiós a los molestos jerjeles.

10. Cupp EW, Cupp MS. Black fly (Diptera simuliidae) salivary secretions: importance in vector competence and disease. J Med Entomol 1997; 34(2): 87-94.

11. Chagas AC, Medeiros JF, Astolfi-Filho S. Anticoagulant activity in salivary gland homogenates of thirspelma guianense (Diptera simuliidae) the primary vector of onchocerciosis in the Brazilian Amazon. Mem Inst Osvaldo Cruz 2010; 105(2): 174-8.
12. Chagas AC, Calvo E, Pimenta PF, Ribeiro JM. An insight into the sialome of simulium guianense, the main vector of River Blindness disease in Brazil. BMC Genomics 2011; 12(1): 612-5.

13. BALlenger JoHn J. Diseases of external ear. En: Diseases of the Nose, Throat, Ear, Head and Neck. Philadelphia:Lea \& Febiger, 1985; $1084-$ 98.

14. Warner Giles, Burgess Andrea, Patel Suresh, Martinez-Devesa Pablo, Corbridge Rogan. Infection and inflammation of the pinna. En: Otolaryngology and Head and Neck Surgery Oxford: Oxford University Press, 2009; 326-9.

15. Gil-Carcedo García LM, Vallejo Valdezate LA, GilCarcedo Sañudo E, López Azanza. Patología del oído externo En: C Suárez, Gil-Carcedo LM, Marco J, Medina JE, Ortega P, Trinidad J. Tratado de Otorrinolaringología y Cirugía de Cabeza y Cuello. Madrid: Editorial Médica Panamericana S.A. 2007: 1341-72.

16. Browning George. Conditions of the pinna and external auditory canal. En: Scott-Brown's Otorhinolaryngology, Head and Neck Surgery $7^{\text {th }}$ edition. London: Hodder Education an Hachette UK Co. 2008; 3321-82.

17. Shin Jennifer, Hartnick Christipher, Randolph Gregory. Evidence Based Otolaryngology. Boston. Springer Science \& Bussiness Media 2008; 3-12. 